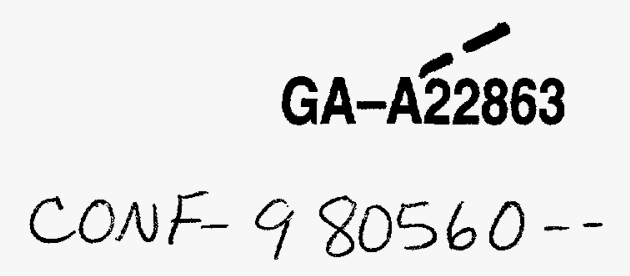

PLASMA PRESSURE AND FLOWS DURING DIVERTOR DETACHMENT by M.J. SCHAFFER, J.A. BOEDO, N.H. BROOKS, R.C. ISLER, O S T I and R.A. MOYER

RECEIVED SEP 281998

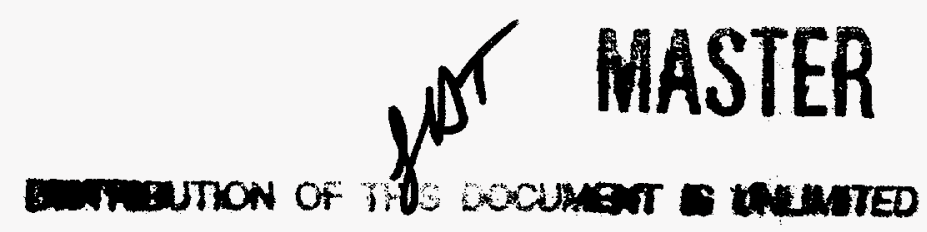

AUGUST 1998 


\section{DISCLAIMER}

Portions of this document may be illegible in electronic image products. Images are produced from the best available original document. 


\title{
PLASMA PRESSURE AND FLOWS DURING DIVERTOR DETACHMENT
}

\author{
by \\ M.J. SCHAFFER, J.A. BOEDO, † N.H. BROOKS, R.C. ISLER, ${ }^{\dagger}$ \\ and R.A. MOYER ${ }^{\dagger}$
}

This is a preprint of a paper to be presented at the 13th International Conference on Plasma Surface Interactions in Controlled Fusion Devices, May 18-23, 1998, San Diego, California and to be published in Journal of Nuclear Materials.

tUniversity of California, San Diego ¥Oak Ridge National Laboratory

\author{
Work supported by \\ the U.S. Department of Energy \\ under Contracts DE-AC03-89ER51114, DE-AC05-96-OR22464, \\ and Grant DE-FG03-95ER54294
}

GA PROJECT 3466

AUGUST 1998 


\section{ABSTRACT}

MHD theory applied to tokamak plasma scrape-off layer (SOL) equilibria requires PfirschSchlüter current, which, because the magnetic lines are open, normally closes through electrically conducting divertor or limiter components. During detached divertor operation the Pfirsch-Schlüter current path to the divertor target is sometimes blocked, in which case theory predicts that the plasma develops a poloidal pressure gradient around the upstream SOL and a corresponding parallel flow, in order to satisfy all the conditions of MHD equilibrium. This paper reports the only known examples of detached diverted plasma in the DIII-D tokamak with blocked Pfirsch-Schlüter current, and they show no clear SOL poloidal pressure differences. However, the predicted pressure differences are small, near the limit of detectability with the available diagnostics. In the more usual DIII-D "partially detached divertor" operation mode, the Pfirsch-Schlüter current appears to never be blocked, and no unusual poloidal pressure differences are observed, as expected. Finally, a local overpressure is observed just inside the magnetic separatrix near the $\mathrm{X}$-point in both attached and detached Ohmically heated plasmas. 


\section{INTRODUCTION}

Pfirsch-Schlüter current arises in all toroidal magnetically confined plasmas to satisfy $\nabla \cdot \mathbf{J}=0$. Pfirsch-Schlüter current in the typical tokamak diverted scrape-off layer (SOL), terminated at each end by an electrically conducting divertor target, passes freely to and through the targets [1]. Target-mounted Langmuir probes detect this current [1,2]. This current can be understood in terms of poloidal equilibrium. Although SOL plasmas stream in the parallel (to B) direction, they do not stream freely in the poloidal direction. They are magnetically confined in both the normal (to the magnetic surfaces) and poloidal directions-a "cross-field-static" equilibrium. The SOL poloidal pressure gradients, which are localized in front of the targets in attached divertor plasmas, are equilibrated by currents crossing the toroidal magnetic magnetic field normal to the magnetic surfaces. See Fig. 1. The Pfirsch-Schlüter currents flow parallel to $\mathbf{B}$ (force-free) to the target, to redistribute current from the high- $B$ side to the low $-B$ side, where extra cross $-B$ current is needed to maintain equilibrium in the weaker magnetic field [1].

However, as detachment is approached the measured target current sometimes disappears $[1,3,4]$. Then, $\nabla \cdot \mathbf{J}=0$ must be satisfied completely within the SOL, in which case previous theoretical work $[3,4]$ requires at least one zone of cross $-B$ current and a corresponding poloidal pressure gradient in the upstream SOL. The additional pressure gradient would modify parallel flow, affecting energy and particle convection, possibly in unanticipated and important ways. The expected pressure differentials in the DIII-D tokamak might be as high as 1.5:1.

The principal goal of this paper is to compare electron pressures $p_{E}$ measured at the inner and outer divertor legs and upstream on a given magnetic surface, in order to test the theoretical prediction of substantial pressure differences. Ion pressures and plasma velocities will be compared in the future if the necessary analysis techniques are validated. The data studied came from experiments run for other purposes, but that had good two-dimensional divertor data both above and below the $\mathrm{X}$-point. Unexpectedly, however, a local electron over pressure was found just inside the magnetic separatrix near the $\mathrm{X}$-point in Ohmically heated plasmas. 


\section{MAGNETIC EQUILIBRIUM THEORY}

Simplified multispecies MHD equilibrium equations with flow were derived in [1] in a form useful for open and closed axisymmetric $(\partial / \partial \phi=0)$ nested toroidal magnetic surfaces. Cylindrical coordinates are $(r, \phi, z)$. Unit vectors $\mathbf{e}_{n}$ and $\mathbf{e}_{p}$ point in the normal and poloidal directions as defined in Fig. 1. The Pfirsch-Schlüter currents are linked to the poloidal component of the momentum equation and $\nabla \cdot \mathbf{J}=0$. In the tokamak limit the poloidal inertial terms are negligible [1], and poloidal momentum conservation reduces to a static pressure balance,

$$
-\nabla_{p} p=J_{n} B_{\phi}=\left(B_{\phi} / r\right) \nabla_{p} \mathfrak{I},
$$

where $\nabla_{p}=\mathbf{e}_{p} \cdot \nabla$ and $\nabla_{n}=\mathbf{e}_{n} \cdot \nabla$. Equation (1) states that any poloidal pressure gradient is equilibrated by current crossing the toroidal magnetic field in the normal direction. The current stream function, $\mathfrak{I}(r, z)=r B_{\phi} / \mu_{0}$, is the poloidal current per toroidal radian, and its gradient yields the current in the poloidal plane:

$$
J_{n}=r^{-1} \mathbf{e}_{p} \cdot \nabla \mathfrak{I}, \quad J_{p}=-r^{-1} \mathbf{e}_{n} \cdot \nabla \mathfrak{I}
$$

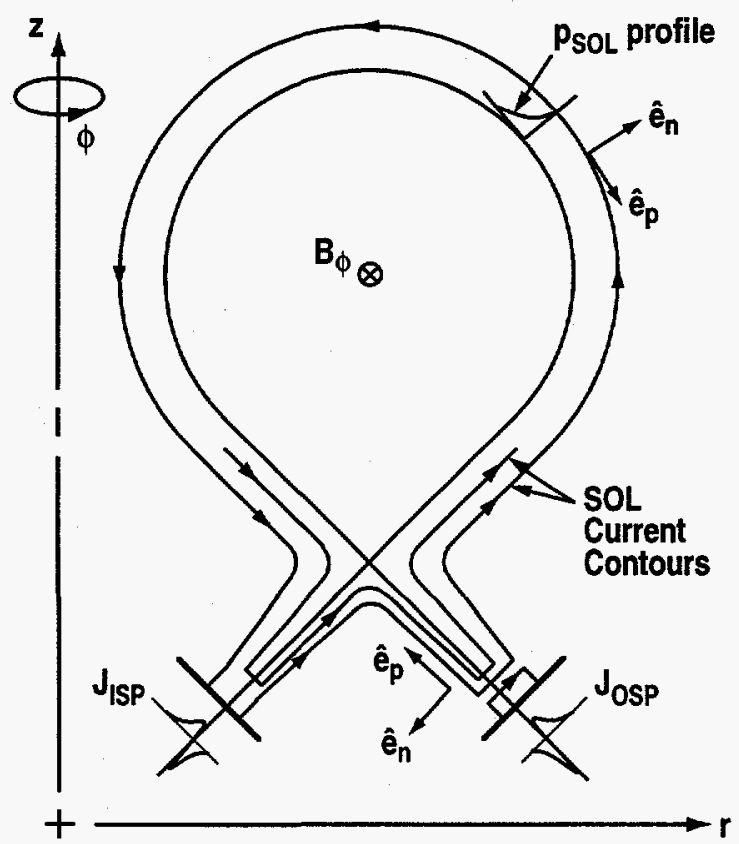

Fig. I. Schematic of normal and poloidal current in a single-null-diverted tokamak. In general current flows to both inner and outer targets, as illustrated, but flow to just one target is also permitted. 
Equations (1) and (2) are further simplified if the poloidal pressure changes occur in poloidal steps and the $J_{n}$ are approximated as current sheets, $I_{n} \equiv \Delta \mathfrak{I}=\int r J_{n} d x_{p}$ [3,4]. Then, for a pressure jump $\Delta p$ at radius $r$, the equilibrium condition is

$$
\Delta p=-\left(B_{\phi} I_{n} / r\right) \approx-R_{0} B_{0} I_{n} / r^{2} .
$$

In attached divertor equilibria, the difference between the upstream SOL pressure $p_{\text {ups }}$ and plasma pressure at the inner and outer target sheaths (much smaller, approximately zero) is equilibrated by the cross $-B$ normal currents $\left|I_{\text {in,out }}\right| \approx p_{\text {ups }} R_{\text {in,out }}^{2} / R_{0} B_{0}$ at target intercept radii $R_{\mathrm{in}, \text { out }}$. The effect of toroidicity is to require unequal normal currents, proportional to $R_{\mathrm{in}, \mathrm{out}}^{2}$, in order to equilibrate the same upstream pressure. The $\nabla \cdot \mathbf{J}=0$ condition is preserved, despite the unequal normal currents, by parallel, force-free Pfirsch-Schlüter currents to the target, as in Fig. 1.

The case of detached divertor plasmas with blocked Pfirsch-Schlüter currents to the target is illustrated in Fig. 2. Now $\nabla \cdot \mathbf{J}=0$ requires $\sum_{i} I_{n_{i}}=0$ on each elementary SOL layer. This is possible, consistent with Eq. (3), only if there are at least two different upstream pressures. These are $p_{\text {in }}$ and $p_{\mathrm{out}}$ in Fig. 2, and there is a corresponding normal current upstream, $I_{\text {ups }}=\left(p_{\text {in }}-p_{\text {out }}\right) R_{\text {ups }}^{2} / R_{0} B_{0}$. In the absence of currents to the target, $\nabla \cdot \mathbf{J}=0$ becomes $I_{\text {out }}+I_{\text {ups }}-I_{\text {in }}=0$. Then

$$
\frac{p_{\text {out }}-p_{\text {in }}}{\left(p_{\text {out }}+p_{\text {in }}\right) / 2}=\frac{R_{\text {out }}^{2}-R_{\text {in }}^{2}}{\mathrm{R}_{\text {ups }}^{2}-\left(R_{\text {out }}^{2}+R_{\text {in }}^{2}\right) / 2}
$$

It is apparent from Eq. (4) that $p_{\text {out }} \neq p_{\text {in }}$ unless $R_{\text {out }}=R_{\text {in }}$, which can happen if e.g. both divertor targets are on the inner wall or outer wall [5]. For conventional geometry, as in Fig. 2, if $R_{\text {ups }}<R_{\text {in }}$, then $I_{\text {ups }}$ crosses a larger $B_{\phi}$ than $I_{\text {in }}$ and $I_{\text {out }}$. Therefore, $I_{\text {ups }}$ equilibrates pressure more effectively and supplements $I_{\text {out }}$. The resulting equilibrium has $p_{\text {in }}>p_{\text {out }}$. If instead $R_{\text {ups }}>$ $R_{\text {out }}$, then $I_{\text {ups }}$ is less effective than even $I_{\text {out }}$. In this case $I_{\text {ups }}$ flows in the same direction as $I_{\text {in }}$ and reduces the overall effectiveness of the total current $I_{\text {in }}+I_{\text {ups }}$ in this direction. In this case $p_{\text {out }}>p_{\text {in. }}$. There are no physical solutions for $R_{\text {in }}<R_{\text {ups }}<R_{\text {out }}$.

Equations (1) and (2) can be used to show similar, more general results for distributed currents. 


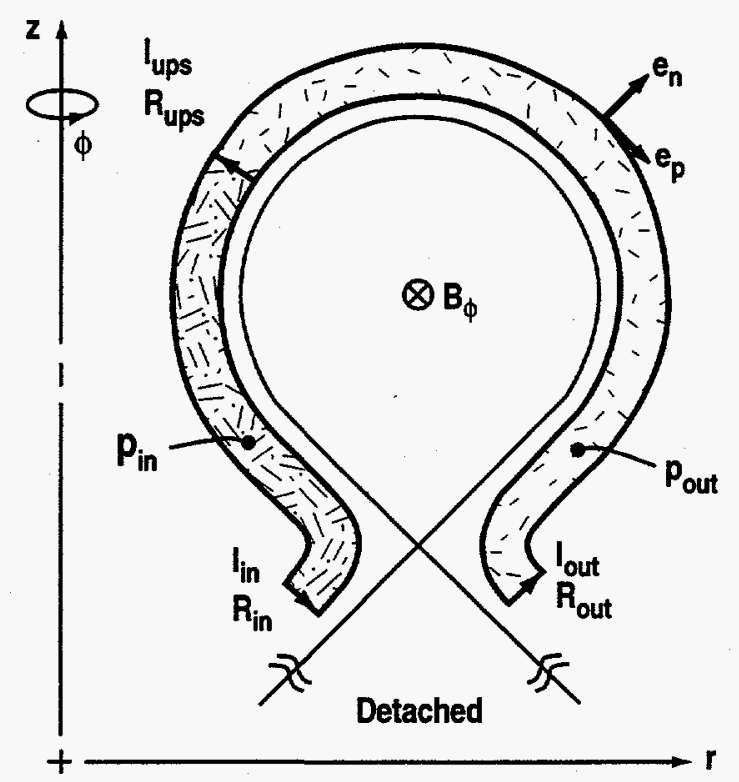

Fig. 2. An elementary SOL plasma layer in a single-null-diverted tokamak. Illustrated are two pressure regions separated by the upstream normal current $I_{\text {ups }}$ as during detachment with blocked Pfirsch-Schlüter currents to the targets. 


\section{EXPERIMENTAL DATA}

Divertor data were taken in two dimensions $(r, z)$ by moving ("sweeping") the divertor magnetically across the fixed diagnostic positions and view chords. An array of fixed Langmuir probes in the divertor target measured the ion saturation current, $I_{\text {sat }}$, and the natural (unbiased) current from the plasma to the target, $I_{\text {nat }}$. The latter contains the combined thermoelectric and Pfirsch-Schlüter currents. Divertor $n_{e}$ and $T_{e}$ were measured by both Thomson scattering at 20 pulses/s [6] and a fast-stroke insertable Langmuir probe [7-10]. In attached plasmas the two techniques agree quite well, but in detached plasmas the Langmuir probe yields temperatures that are far too high [9,10]. Only Thomson scattering data are presented in this paper. The insertable divertor probe array also measured parallel velocity, by a Mach probe pair [10], and plasma potential, from which the perpendicular electric field is derived. Additional diagnostics since late 1997 will permit measurement of divertor $T_{i}$ and parallel velocity by Doppler broadened and shifted visible spectroscopic lines along multiple viewing chords [11]. In the upstream plasma $n_{e}$ and $T_{e}$ were measured by Thomson scattering at 140 pulses/s, and $T_{i}$ is measured by charge exchange recombination Doppler spectroscopy when neutral beams are used. Core plasma profiles are stationary during the divertor sweeps.

We present data from three interesting shots. The tokamak discharges were magnetically diverted with a lower single poloidal field null. Plasma current was 1.3 or $1.4 \mathrm{MA}$, and the toroidal magnetic field was $2.1 \mathrm{~T}$ with the ion $\operatorname{grad} B$ drift downward, toward the $\mathrm{X}$-point. Figure 3 shows shot 87527 at the beginning, middle and end of a continuous divertor geometry sweep. Divertor and upstream Thomson scattering points are also shown. The insertable probe moves along the same line as the divertor Thomson points.

A critical part of the pressure comparison is to accurately map the divertor and upstream data onto magnetic surfaces. The magnetic surfaces were generated at the time of each divertor Thomson scattering measurement by the equilibrium reconstruction code EFIT [12]. There is some uncertainty in the reconstruction, typically $\pm 1 \%-2 \%$ in $\tilde{\psi}$, the normalized poloidal flux ( $\tilde{\psi}=1$ at separatrix). Therefore, the upstream magnetic surfaces were adjusted, where necessary, to make $T_{e}$ appear constant along surfaces slightly inside the magnetic separatrix.

The first shot is 87522, an attached Ohmic plasma, with both inner and outer strike point plasmas attached, as determined by measured $I_{\text {sat }}$ and $T_{e}$ at the target. Approximately 10$20 \mathrm{~A} /$ (toroidal radian) of Pfirsch-Schlüter current was measured at the targets, in agreement with theoretical values of 10-20 A/rad. Figure 4 shows overplots of divertor (inner and outer legs) and upstream $p_{e}, n_{e}$ and $T_{e}$, measured by Thomson scattering, as a function of $\tilde{\psi}$ for this shot. The large $T_{e}$ and $n_{e}$ scatter are due much more to plasma fluctuations than to measurement error. The 

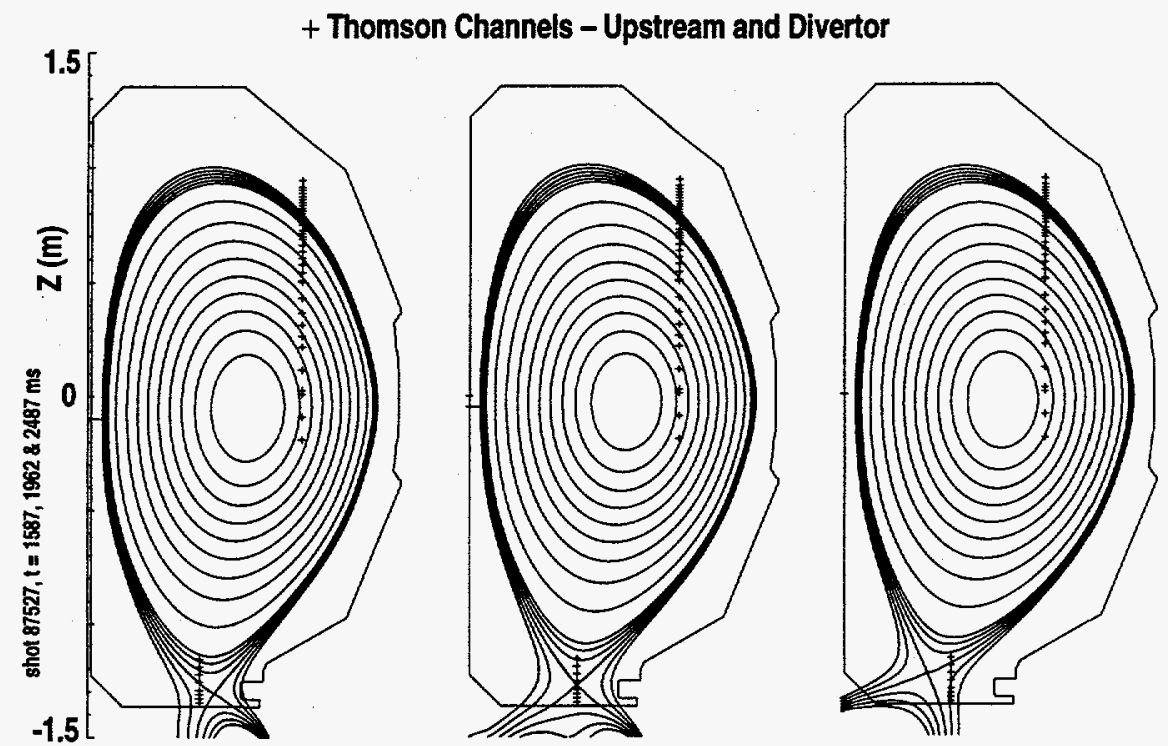

Fig. 3. Illustrating the range of divertor sweep across the eight divertor Thomson scattering view points (crosses) at $R=1.48 \mathrm{~m}$. The highest view point is $21 \mathrm{~cm}$ above the target. Upstream Thomson scattering view points (crosses) at $R=1.94 \mathrm{~m}$ are also shown. Note the relatively low separatrix $X$-point, needed to make measurements above the $X$-point.

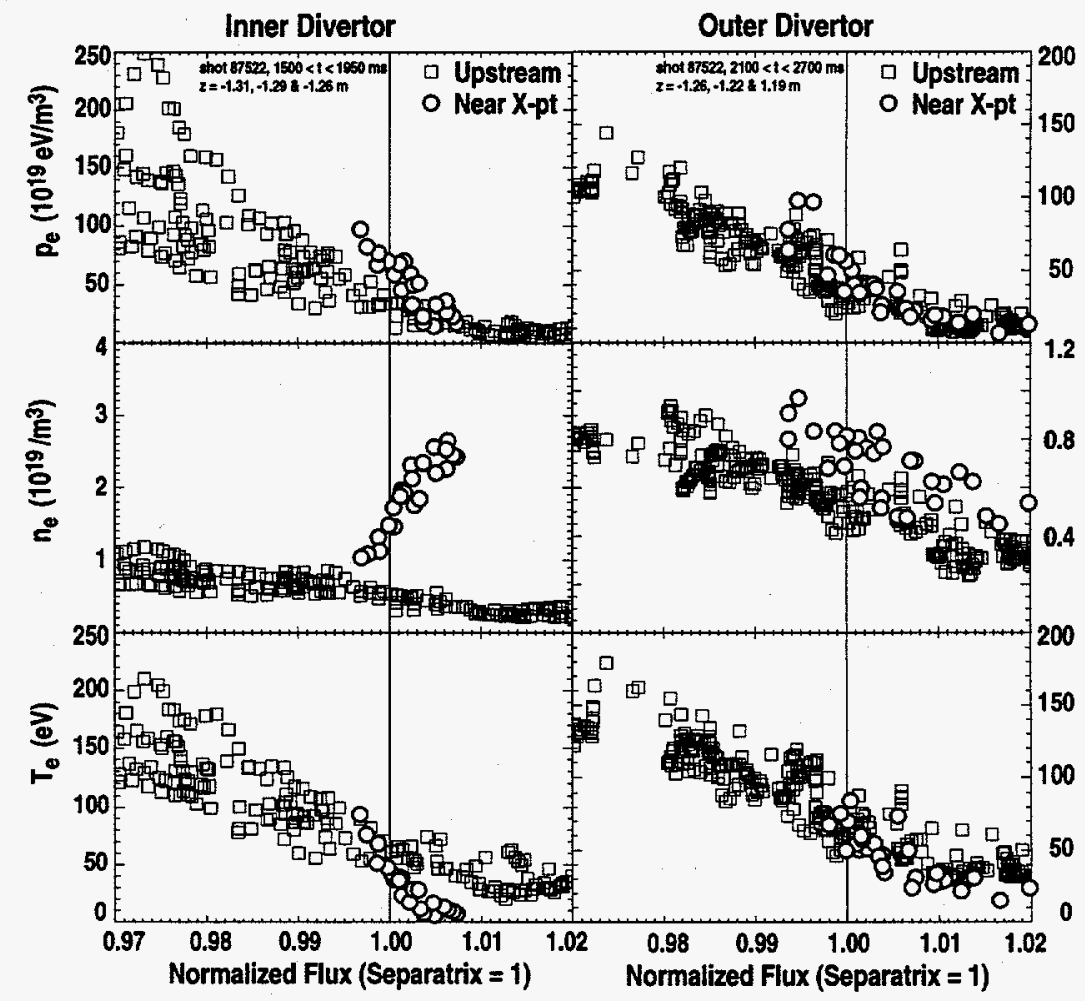

Fig. 4. Upstream (squares) and divertor (circles) $p_{e}, n_{e}$ and $T_{e}$ versus normalized poloidal magnetic flux, from attached Ohmic shot 87522. Inner divertor data are from Thomson scattering points between 5 and $11 \mathrm{~cm}$ above the target and $1550<t<1950 \mathrm{~ms}$. Outer data are from points between 9 and $17 \mathrm{~cm}$ above the target and $2100<t$ $<2700 \mathrm{~ms}$. 
space ( $\Delta \tilde{\psi} \approx 0.013$ ) between the discrete upstream Thomson points get filled by the small uncontrolled motion of the upstream plasma across the fixed viewing points. The figure shows a small overpressure on the inner SOL with respect to the upstream pressure, which is surprising, because overpressure is not expected with attached divertors. The apparent SOL overpressure can be eliminated by a displacement of the inner SOL data, by $\Delta \tilde{\psi} \approx 0.002$, which serves to illustrate the sensitivity of the profile alignments to small magnetic reconstruction errors.

Figure 5 shows similar overplots for shot 87527. This Ohmic plasma detached from both the inner and outer targets during strong deuterium gas puffing. The detachment was characterized by $T_{e} \leq 2 \mathrm{eV}$ everywhere at the targets and almost complete elimination of target heating, but $I_{\text {sat }}$ remained large and $p_{e}$ was only somewhat reduced at the targets. A Pfirsch-Schlüter current of $\sim 2 \mathrm{~A} / \mathrm{rad}$, not much larger than the measurement limit of $I_{\text {nat }}$, was present only briefly during detachment. The theoretical Pfirsch-Schlüter current was $\sim 20 \mathrm{~A} / \mathrm{rad}$. Therefore, the PfirschSchlüter target current was blocked in this shot. The figure shows a possible slight overpressure on the outer SOL. The divertor data plotted are from slightly above the height of the X-point. At lower heights, $p_{e}$ was smaller. The higher Thomson scattering view points are all on closed magnetic surfaces, so the SOL pressure is not known farther above the divertor. It might seem that the data contradict the theory. However, the expected pressure difference is not large. Calculation from Eq. (4) with $R_{\text {in }}=1.35 \mathrm{~m}, R_{\text {out }}=1.47 \mathrm{~m}, R_{\text {ups }}=2.26 \mathrm{~m}$ (separatrix outermost radius) yields

$$
\frac{p_{\text {out }}-p_{\text {in }}}{\left(p_{\text {out }}+p_{\text {in }}\right) / 2} \approx+0.11,\left(p_{\text {out }}>p_{\text {in }}\right),
$$

which is too small to see reliably in the available data. It is not known how the plasma might choose its upstream poloidal pressure gradient radius $R_{\text {ups }}$, but it is a plausible hypothesis that it would do so in a way that would least perturb the SOL pressure and flow. Our choice of $R_{\text {ups }}$ for the estimate of Eq. (5) gives the smallest possible pressure ratio from Eq. (4). Equation (4) is from a pressure jump model. If the pressure gradient were gradual and distributed more generally around the magnetic surface, then $p_{\text {out }}-p_{\text {in }}$ in the DIII-D observable region near the X-point could in principle be lower, even zero, but the peak pressure difference elsewhere on the surface would be greater.

It might be argued that large neutral gas pressure in the divertor can equilibrate the expected $p_{\text {out }}-p_{\text {in }}$. Although neutral pressure is neglected in Eq. (4), it is retained in the full theory [1], where it simply adds to the plasma pressure in the poloidal momentum equation. The measured neutral gas pressure in shot $87527, \sim 0.4 \mathrm{~Pa}$, is very much less than the SOL plasma pressure, where $p_{e}$ alone is $\sim 50 \mathrm{~Pa}$. Therefore, neutral pressure alone did not affect the plasma pressure. 


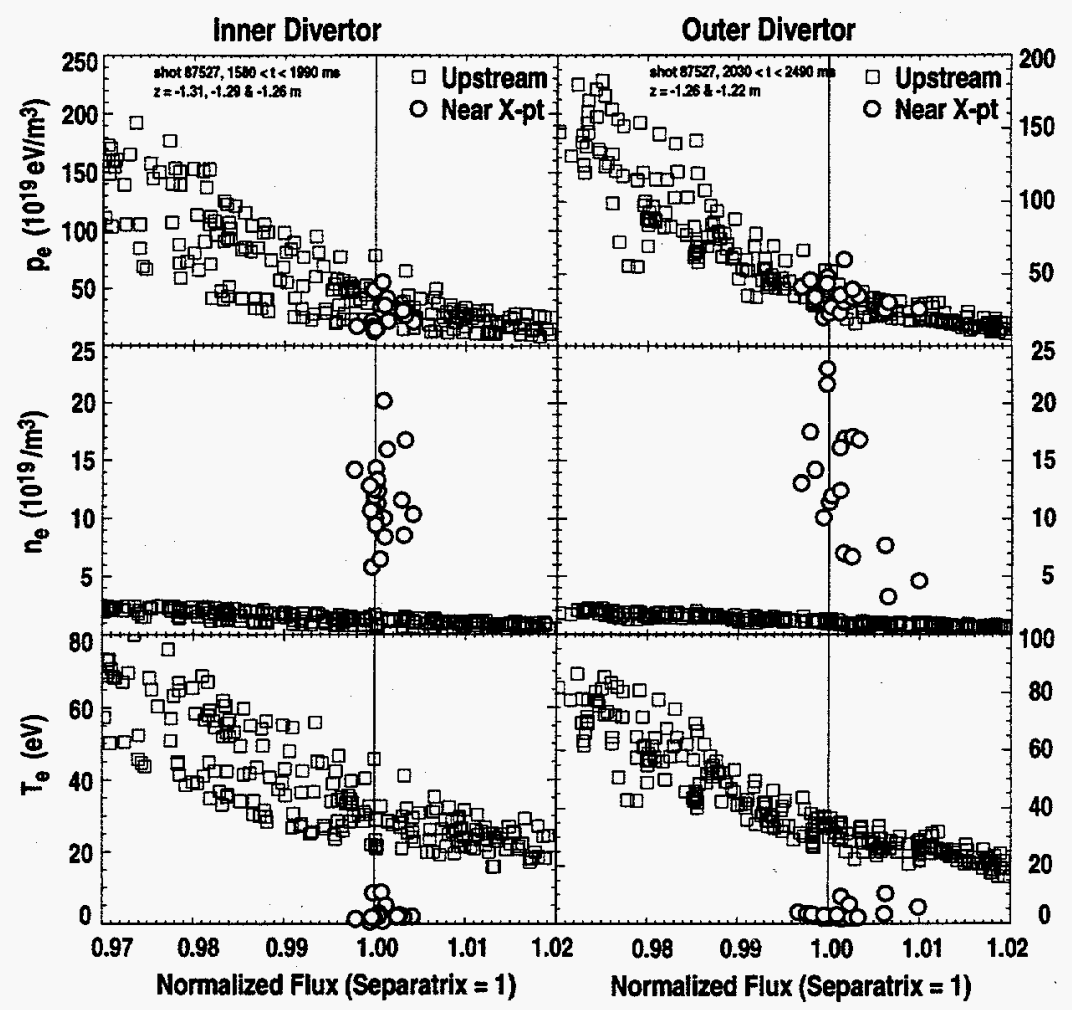

Fig. 5. Plots as in Fig. 4, but from detached Ohmic shot 87527. Inner divertor data are from Thomson scattering points between 5 and $11 \mathrm{~cm}$ above the target and $1580<t<1990 \mathrm{~ms}$. Outer data are from points between 11 and $14 \mathrm{~cm}$ above the target and $2030<t<2490 \mathrm{~ms}$.

Figure 6 shows $p_{e}, n_{e}$ and $T_{e}$ for shot 94007 . This rapidly ELMing plasma displays the classic features of a partially detached divertor (PDD) [13]: $T_{e} \leq 2 \mathrm{eV}$ everywhere at the targets, and reduced $p_{e}$ and target heating near the separatrix, but not farther out. The divertor sweep did not bring the inner leg into diagnostic view, and the Figure shows divertor data only from the outer leg and on closed surfaces above the X-point. Despite the "partial" detachment, the Pfirsch-Schlüter current $(\sim 50 \mathrm{~A} / \mathrm{m})$ is not blocked in this shot, and there is always ample $I_{\text {sat }}$ $(>250 \mathrm{~A} / \mathrm{rad})$ to carry it. Within the data scatter there does not appear to be any divertor overpressure, which is consistent with the presence of target Pfirsch-Schlüter current. Furthermore, preliminary and incomplete divertor velocity data from similar PDD plasmas show no flow features that can not be explained conventionally, which is again consistent with the presence of target Pfirsch-Schlüter current.

In Fig. 7, the $p_{e}, n_{e}$ and $T_{e}$ on closed magnetic surfaces just above the X-point are compared with their upstream counterparts, for both the attached (87522) and detached (87527) Ohmic shots. Both shots exhibit overpressure above the X-point, an unexpected occurrence. The electron overpressure is associated with large local plasma density, which is presumably generated by recycling neutrals entering the plasma from the divertor private flux region below. 


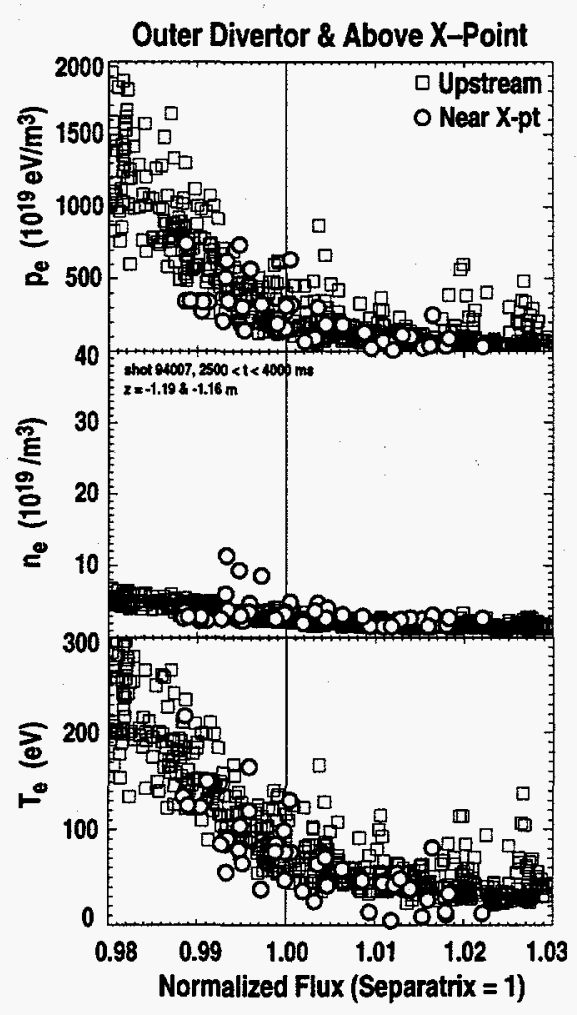

Fig. 6. Plots as in Fig. 4, but from partially detached ELMy H-mode shot 94007 . The divertor sweep did not bring inner SOL points into diagnostic view. Data are from Thomson scattering points between 17 and $21 \mathrm{~cm}$ above the target and $2500<t<4500$ ms. Points at normalized flux $<1$ lie on closed magnetic surfaces above the X-point. ELM-affected data are excluded.

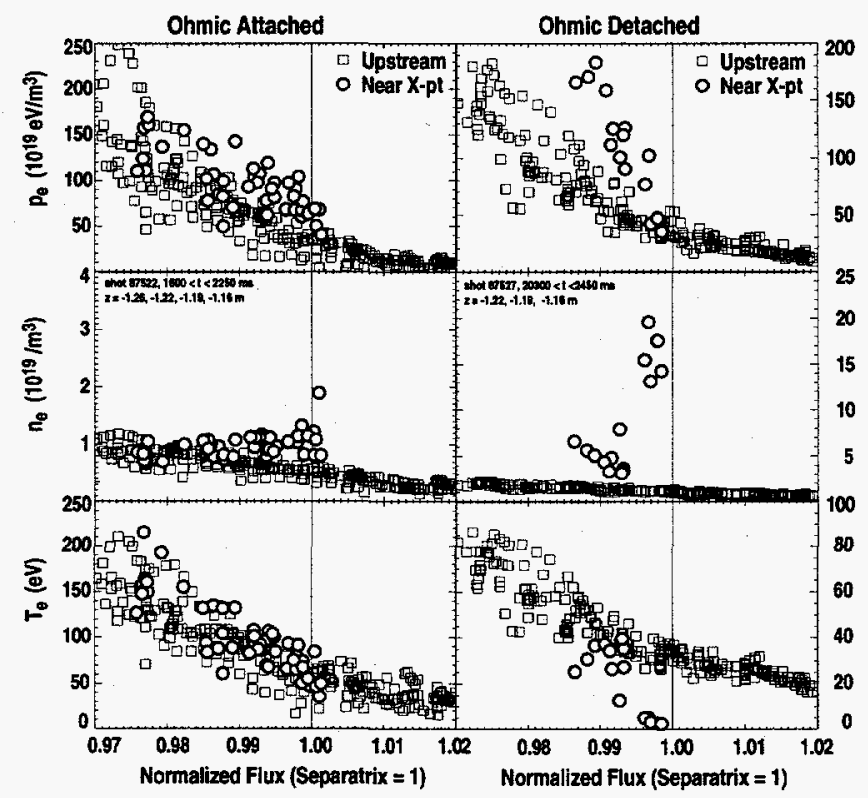

Fig. 7. Plots as in Fig. 4, but from closed magnetic surfaces above the X-point. Data are from attached and detached Ohmic shots, 87522 and 87527, respectively. Thomson scattering points are between 11 and $21 \mathrm{~cm}$ in the former shot and between 14 and $21 \mathrm{~cm}$ in the latter. 
However, since ion temperature is usually higher than $T_{e}$ upstream, at the midplane, it is possible that the total pressure is constant along the magnetic lines. The electron overpressure disappears farther in, where $n_{e}$ and $T_{e}$ are equilibrated on the surfaces. If the observed overpressures are real, they should drive parallel plasma flows away from the $\mathrm{X}$-point. However, there are no ion temperature nor divertor velocity data from these 1995 shots to test this prediction and add confidence to the overpressure observation. Future experiments are needed to verify and investigate this phenomenon. 


\section{CONCLUSION}

The data show that not all "detached" divertor modes necessarily lead to blocked PfirschSchlüter current. In the most common and most studied DIII-D detached divertor mode, the partially detached divertor (PDD) with ELMy H-mode, the Pfirsch-Schlüter current was not blocked, and no abnormal pressure gradients were observed in the PDD divertor region, in agreement with the theory.

We found only two examples of a detached diverted DIII-D plasma with blocked PfirschSchlüter current. These were Ohmically heated plasmas. Despite the absence of Pfirsch-Schlüter current, there were no clear signs of pressure differences between the plasmas just above the divertor pressure gradients and the upstream plasma. This finding appears to contradict the theoretical prediction. However, this conclusion is not definitive, because the theoretically predicted magnitude of the in-out pressure difference is on the order of the random and systematic experimental uncertainties. Also, to date we have only analyzed electron pressures, but the theoretical prediction applies to total pressure. Ion pressures are already measured upstream in DIII-D, but techniques are still being developed to calculate divertor ion pressure accurately from multi-chordal Doppler broadened optical emission lines.

An unexpected electron overpressure was observed on closed magnetic surfaces just inside the magnetic separatrix, near the $\mathrm{X}$-point in both attached and detached Ohmically heated plasmas.

It is difficult to observe poloidal pressure gradients in a diverted tokamak SOL. Very accurately mapped magnetic surfaces are required to avoid systematic errors. Large pressure fluctuations commonly present in SOL plasmas mean that many data must be averaged to make the confidence interval consistent with the expected equilibrium pressure effect. Sweeping the plasma across fixed one-dimensional diagnostic views, as was done here, introduces the possiblity that the plasma changes in the course of the extreme divertor geometry changes. Finally, it is difficult to get a sufficiently high and wide diagnostic view of the divertor plasma in typical tokamaks. 


\section{REFERENCES}

[1] M.J. Schaffer, A.V. Chankin et al., Nucl. Fusion 37 (1997) 83.

[2] D. Buchenaur et al., J. Nucl. Mater. 176-177 (1990) 528.

[3] M.J. Schaffer, "Non Uniform Poloidal Pressure is Necessary for Detached Tokamak Divertor Operation," Comments Plasma Phys. Controlled Fusion 19 (1998) to be published.

[4] M.J. Schaffer, Contrib. Plasma Phys. 38 (1998) 140.

[5] M.J. Schaffer et al., Bull. Am. Phys. Soc. 40 (1995) 1832.

[6] T.N. Carlstrom et al., Rev. Sci. Instrum. 68 (1997) 1195.

[7] J.G. Watkins et al., Rev. Sci. Instrum. 68 (1997) 373.

[8] R.A. Moyer et al., this conference.

[9] R.A. Moyer et al., J. Nucl. Mater. 241-243 (1997) 633.

[10] J. Boedo et al., this conference.

[11] R.C. Isler, N.H. Brooks et al., this conference.

[12] L.L. Lao et al., Nucl. Fusion 31 (1985) 1111.

[13] T.W. Petrie et al., Nucl. Fusion 37 (1997) 321. 


\section{ACKNOWLEDGMENT}

This work was supported by the U.S. Department of Energy under Contracts DE-AC0389ER51114, DE-AC05-OR22464, and Grant No. DE-FG03-95ER54294. 